\title{
Effects of Dorsal Hippocampal Damage on Conditioning and Conditioned-response Timing: A Pooled Analysis
}

Shu K.E. Tam ${ }^{1, *}$, Dómhnall J. Jennings ${ }^{2}$ and Charlotte Bonardi ${ }^{3}$

${ }^{1}$ Nuffield Laboratory of Ophthalmology, University of Oxford, Level 5-6 West Wing, John Radcliffe Hospital, Headley Way, Oxford, OX3 9DU, United Kingdom ${ }^{2}$ Institute of Neuroscience, Henry Wellcome Building, Newcastle University, Framlington Place, Newcastle upon Tyne, NE2 4HH, United Kingdom ${ }^{3}$ School of Psychology, University of Nottingham, University Park, Nottingham, NG7 2RD, United Kingdom

Word count in main text: 8663

Number of tables: 4

Number of figures: 9

Number of supplementary material: 1

Running title: Hippocampus and response timing

Keywords: Pavlovian conditioning; interval timing; peak procedure

"Correspondence to: Shu K. E. Tam (tel: +44 7854596500; e-mail: eric.tam@ndcn.ox.ac.uk), Nuffield Laboratory of Ophthalmology, University of Oxford, Level 5-6 West Wing, John Radcliffe Hospital, Headley Way, Oxford, OX3 9DU, United Kingdom 


\begin{abstract}
Behavioral findings suggest that the dorsal hippocampus (DHPC) plays a role in timing of appetitive conditioned responding. The present article explored the relationship between the extent of DHPC damage and timing ability, in a pooled analysis of three published studies from our laboratory. Initial analyses of variance confirmed our previous reports that DHPC damage reduced peak times (a measure of timing accuracy). However, the spread (a measure of timing precision) was unchanged, such that the coefficient of variation (spread / peak time) was significantly larger in DHPC-lesioned animals. This implies that, in addition to the wellestablished effect of DHPC lesions on timing accuracy, DHPC damage also produced a deficit in precision of timing. To complement this analysis, different generalized linear mixed-effects models (GLMMs) were performed on the combined dataset, to examine which combinations of the different behavioral measures of timing were the best predictors of the degree of hippocampal damage. The results from the GLMM analysis suggested that the greater the DHPC damage, the greater the absolute difference between the observed peak time and reinforced duration. Nevertheless, this systematic relationship between damage and performance was not specific to the temporal domain: paradoxically the greater the damage the greater the magnitude of peak responding. We discuss these lesion effects in terms of scalar timing theory.
\end{abstract}




\section{Introduction}

There is now compelling behavioral and electrophysiological evidence that the hippocampus is involved in spatial cognition, and moreover, it is the dorsal hippocampus (DHPC) that is crucial. For example, Moser et al. (1995) systematically manipulated the volume of hippocampal damage, starting from either the dorsal or ventral pole of the structure. They confirmed that the DHPC, but not the ventral hippocampus (VHPC), was critical for navigation to a hidden goal using distal visual cues, and that there was a positive relationship between the extent of DHPC damage and spatial navigation deficit (see also Moser et al., 1993). Further evidence from single-unit recording studies suggests that, although pyramidal neurons of both the DHPC and VHPC have location-specific receptive fields (place cells), those of the DHPC are more stable, coherent, and location-specific than those of the VHPC (Jung et al., 1994; Kjelstrup et al., 2008; Royer et al., 2010).

Nevertheless, the role of the DHPC in cognition is not confined to the spatial domain, and it may also be involved in temporal aspects of learning or performance (Olton, 1986; Kesner, 1998; Sakata, 2006; Howard \& Eichenbaum, 2013). For example, McEchron et al. (2003) trained rabbits that a fixed-duration, 3-s conditioned stimulus (CS) was paired with a paraorbital shock after an empty, trace interval of either 10 or 20 s. On non-reinforced test trials when the empty trace period was extended, some pyramidal neurons of the DHPC fired maximally at the time point at which the shock had been applied during conditioning. Electrophysiological findings of this type complement earlier reports by Meck et al. (1984), who examined the effect of lesions of the fimbria-fornix (fibres connecting the hippocampus with various subcortical structures) in a similar appetitive instrumental task. A fixed-duration, 20-s stimulus was paired with food at its termination, and subsequently non-reinforced test trials with an extended stimulus period were 
presented. On the test trials, the time point at which maximal appetitive responding (lever pressing) occurred was estimated. In the control animals, maximal responding occurred at the time at which food was delivered on the training trials, but the subjects with fimbria-fornix lesions exhibited maximal responding at earlier time points (see also Meck, 1988).

These findings are consistent with the possibility that the hippocampus, or the DHPC, is involved in temporal cognition; but the behavioral evidence, at least, is not conclusive in this respect. The subjects in the study of Meck et al. (1984) underwent fimbria-fornix lesions which, by damaging fibres connecting the hippocampus with various subcortical structures, could have had different behavioral effects from those produced by damage to the hippocampus itself (e.g., Sanderson et al., 2006 vs. Aggleton et al., 2009). Until recently there was almost no behavioral evidence that could unequivocally establish that damage to the hippocampus, and more specifically the DHPC, is sufficient to produce a temporal deficit (although see Jaldow et al., 1989; Balci et al., 2009; Yin \& Meck, 2014). In order to address this deficiency, we examined the effect of ibotenic-acid lesions of the DHPC in an appetitive conditioning task, in which a fixed-duration, 15-s CS was followed by a food pellet at its termination (Tam \& Bonardi, 2012a; Tam et al., 2013). On subsequent non-reinforced test trials we found that, while the shamlesioned subjects showed maximal responding at the time point at which food had been delivered during training, the subjects with DHPC damage showed maximal responding at significantly earlier time points (i.e. they underestimated the CS duration). Thus, damage to the DHPC is sufficient to reproduce the temporal deficits observed after fimbria-fornix lesions.

However, other results from our laboratory slightly complicate this picture. In a further study, Tam and Bonardi (2012b) examined both conditioned responding and timing to a CS of 40-s duration. They reported that while lesioned subjects showed numerically earlier peak times 
than sham-lesioned animals, this effect was not statistically significant; nevertheless, they did show significantly more absolute error in timing (i.e. peak time - CS duration|). In other words the lesioned subjects overestimated the CS duration, as well as underestimating it. This difference was numerically present, although not statistically significant, in one of the two studies with the shorter CS (Tam et al., 2013).

Taken together these findings suggest that the DHPC plays a role in timing of instrumental and Pavlovian conditioned response. However, the exact relationship between the extent of DHPC neuronal loss and timing performance has never been examined-this was the purpose of the present study. In our earlier reports there was variability in the extent of DHPC damage, and also in both the extent and the precise nature of the timing deficit among the lesioned subjects (Tam \& Bonardi, 2012a,b; Tam et al., 2013). Data of this type thus provide an opportunity to examine the relationship between DHPC damage and response timing in a more quantitative manner, using both actual time of maximal responding (peak time) and absolute error (|peak time - CS duration|) as measures of timing performance.

The present article thus explored the relationship between DHPC damage and timing performance using a statistical approach which employed data from three earlier reports conducted in this laboratory: Tam et al. (2013); Tam and Bonardi (2012a,b), denoted below as Experiments 1, 2, and 3, respectively. Initially the data from the three studies were pooled, and performance was compared in animals with and without DHPC damage. Then a series of generalized linear mixed-effects models was performed (GLMMs; e.g., Crawley, 2007), in order to examine the degree to which the extent of hippocampal damage could be predicted by the different behavioral measures of timing. This approach has two distinct benefits in throwing light on the interaction between DHPC damage and behavioral performance. First, while analysis of 
variance (ANOVA) can only test the null hypothesis that one specific behavioural measure is unaffected by DHPC damage, GLMM has more in common with multiple regression in that it can simultaneously evaluate the relationship between several behavioural measures. Second, we are pooling data from three different experiments that differed in various respects, such as the duration and modality of the CSs. The key relationship between the various behavioural measures and the amount of hippocampal damage might vary under these different experimental conditions. For example, it is well documented that auditory and visual CSs of the same duration are judged as different, an effect which has been interpreted in terms of different clock speeds for different stimulus modalities (e.g., Penney et al., 2000). Critically, GLMM is capable of taking this into account: It permits us to control for the effects of parametric differences among the studies, and so can yield meaningful results in spite of the fact that the studies differed in terms of CS duration, modality, degree of hippocampal damage, etc. (Burnham et al., 2002; 2011; Field et al., 2012). Evidence of a significant relationship between damage and timing performance would strengthen the notion that the DHPC is important for temporal processing (Olton, 1986; Kesner, 1998; Sakata, 2006; Howard \& Eichenbaum, 2013). 


\section{Methods}

\section{Animals}

Seventy-two Lister Hooded male rats were used in all, twenty-four in each of the three studies. In each experiment 12 animals were assigned to the sham-lesioned group and 12 to the DHPC-lesioned group. Five DHPC-lesioned subjects in Experiment 1 (Tam et al., 2013) were excluded from the pooled analyses because their hippocampal damage was unilateral; one DHPC-lesioned subject in Experiment 2 (Tam \& Bonardi, 2012a) was excluded because it did not respond on any of the test trials, resulting in $n=30$ in the DHPC-lesioned group in the pooled analyses. One sham-lesioned subject in Experiment 1 was excluded because some of its coronal sections were lost during the staining process and hence its hippocampal area could not be determined, resulting in $n=35$ in the sham-lesioned group in the pooled analyses. Subjects of the same lesion group were caged in pairs in a colony with a light-dark cycle of $12 \mathrm{hrs;} \mathrm{light}$ phases started at 07:00. The weight of each subject at the start of surgery was approximately 300 g; two weeks after surgery animals were deprived to $85 \%$ of their ad lib-weight, and maintained at this level (with adjustments for natural growth rate) by feeding each subject a restricted daily ration after each experimental session. Subjects were tested seven days a week during each study. At the beginning of the experiments, the average weight of the subjects at the start of each study was, for Experiments 1-3 respectively: 387 g (range: 350-435 g), 307 g (range: 281-344 g), and $375 \mathrm{~g}$ (range: $325-435 \mathrm{~g})$.

\section{Surgery}

Subjects were anaesthetized with isofluorane. The scalp was incized along the midline and the facial muscles were retracted. Portions of cranial bone above the DHPC were removed 
with an electric drill. Dorsal hippocampal lesions were achieved by injecting 0.05 or $0.1 \mu 1$ of ibotenic acid into 14 sites (Table 1). The AP and ML coordinates shown in Table $\mathbf{1}$ were relative to bregma, whereas the DV coordinates were relative to the brain surface. The concentration of the injected ibotenic acid solution was $63 \mathrm{mM}$, which was made from dissolving $5 \mathrm{mg}$ of ibotenic acid solids (Sigma-Aldrich, Dorset, UK) into $0.5 \mathrm{ml}$ of 0.1-M phosphate-buffered saline (pH 7.4). Injections were administered by an infusion pump (KD Scientific, Holliston, Massachusetts) at rates of $0.03 \mu 1 / \mathrm{min}$ using a $2-\mu 1$ syringe (Hamilton, Bonaduz, Switzerland) with a 25-gauge, bevel-tip needle. After each injection the needle was left in situ for $1 \mathrm{~min}$ before it was withdrawn and moved to the next site. Subjects in the sham-lesioned group received similar treatment but no injection of ibotenic acid. After all sites were visited, the scalp was sutured. Subjects were injected subcutaneously with $1 \mathrm{ml} / \mathrm{kg}$ of Rimadyl (Pfizer, Surrey, UK) as analgesic and $0.5 \mathrm{ml}$ of warmed saline to prevent dehydration during surgery, and all of them fully recovered within two weeks.

\section{Apparatus and Stimuli}

Eight operant chambers (Med Associates, St. Albans, Vermont; length $\times$ width $\times$ height: $30 \mathrm{~cm} \times 25 \mathrm{~cm} \times 25 \mathrm{~cm}$ ) were used; each chamber was located inside a sound- and lightattenuating chamber $(72 \mathrm{~cm} \times 32 \mathrm{~cm} \times 42 \mathrm{~cm})$ equipped with a ventilation fan. The ambient sound level inside the operant chamber when the ventilation fan was switched on was $65 \mathrm{~dB}(\mathrm{~A})$. Each operant chamber had two aluminium short walls and two transparent plastic long walls (the front one served as the door). The ceiling was a piece of transparent plastic. The floor consisted of 19 stainless steel bars spaced $1 \mathrm{~cm}$ apart; each had a diameter of $0.5 \mathrm{~cm}$ and ran parallel to the short walls; located below the floor was a pan containing a layer of sawdust bedding which was changed weekly. A recessed food magazine was located on one of the short walls, equidistant 
from the long walls and $3 \mathrm{~cm}$ above the floor. The magazine was accessible via a rectangular aperture (width $\times$ height: $4 \mathrm{~cm} \times 5 \mathrm{~cm}$ ); an infrared beam was sent from one side of the magazine and received on the other side; each interruption of the beam was recorded as a discrete response. The chambers were not illuminated in Experiments 2 and 3; in these two studies the CS was either a white noise or a 1-kHz click of $75 \mathrm{~dB}(\mathrm{~A})$, presented via a speaker located at the upper corner of the short wall, opposite to the wall in which the food magazine was located. In Experiment 1 the CS was a 2.8-W houselight, the bottom half of which was shielded, located 11 $\mathrm{cm}$ above the magazine; when the CS was not presented, the chambers were not illuminated. In all three studies, the unconditioned stimulus (US) was one 45-mg food pellet (PJAI-0045; Noyes, Lancaster, New Hamsphire) delivered into the magazine. Experimental events (delivery of CS and US, and head entry responses) were controlled and recorded by the Med-PC software (version IV) installed on a PC located in another room.

\section{Procedure}

Experiment 1: 15-s Visual Conditioned Stimulus (Tam et al., 2013)

In Experiment 1 subjects were first given a 40-min magazine training session in which USs were delivered according to a variable-time, 240-s schedule. There followed six sessions of acquisition, each containing 64 delay conditioning trials on which the CS was a 15-s houselight, and US delivery occurred at CS termination. The intertrial interval (ITI, the interval between the end of one trial and CS onset on the next) comprized a random interval (drawn from an exponential distribution) with a mean of $60 \mathrm{~s}$ plus a fixed interval of $30 \mathrm{~s}$. Test sessions 7-22 were identical to the acquisition sessions, except that half of the conditioning trials (32 trials) were replaced by the non-reinforced test trials (peak trials), on which the CS lasted for $45 \mathrm{~s}$. 
These peak trials assessed how well the subjects timed from CS onset. The number of magazine entries was recorded in each 1-s bin of the peak trials. Conditioning and peak trials were presented in a randomized order, with the constraint that each session began with a conditioning trial.

Experiment 2: 15-s Auditory Conditioned Stimulus (Tam \& Bonardi, 2012a)

In Experiment 2, after a session of magazine training identical to that in Experiment 1, subjects were given eight sessions of acquisition, each containing eight delay conditioning trials (they also received eight trace conditioning trials in each session, randomly intermixed with the delay conditioning trials, but the data from these trials were not included in the present analyses). The auditory CS was 15 s, and US delivery occurred at CS termination. Six of the DHPC- and sham-lesioned subjects received the click as the CS and the remainder the noise. The ITI comprized a random interval with a mean of $150 \mathrm{~s}$ and a fixed interval of $60 \mathrm{~s}$. After the acquisition phase, subjects were given four sessions of extinction (sessions 9-12), each containing four non-reinforced presentations of the CS (subjects also received an equal number of trace CS extinction trials, randomly intermixed with the delay CS extinction trials in each session). The fixed portion of the ITI was increased to $75 \mathrm{~s}$, but in all other respects these sessions were identical to the acquisition sessions. At this point the animals received some unrelated appetitive training with visual CSs, before retraining sessions 13 and 14 took place, which were identical to sessions 1-8. Test sessions 15-42 were identical to the acquisition sessions, except that each session contained an additional, non-reinforced peak trial, on which the delay CS was presented for $45 \mathrm{~s}$ (each session also contained one non-reinforced peak trial of the trace CS); programming issues allow us to present the data from sessions 23-42 only. The ITI was a random interval with a mean of $120 \mathrm{~s}$ plus a fixed interval of $90 \mathrm{~s}$. 
Experiment 3: 40-s Auditory Conditioned Stimulus (Tam \& Bonardi, 2012b)

In Experiment 3, after a session of magazine training, subjects were given 10 sessions of acquisition, each containing 50 delay conditioning trials. The auditory CS was $40 \mathrm{~s}$ in duration, and US delivery occurred at CS termination; the identity of the CS (click or noise) was counterbalanced as in Experiment 2. The ITI comprized a random interval with a mean of $40 \mathrm{~s}$ and a fixed interval of $40 \mathrm{~s}$. Test sessions 11-40 were identical to the acquisition sessions, except that 15 of the 50 conditioning trials were randomly replaced by the non-reinforced peak trials, on which the CS lasted for $80 \mathrm{~s}$.

\section{Histology}

Subjects were sacrificed with an overdose of pentobarbitone and perfused intracardially with formal saline. Their brains were stored in formal saline at room temperature for two days, subsequently in $20 \%$ sucrose solution at a temperature of $4{ }^{\circ} \mathrm{C}$ for two days. Brains were then cut with a cryostat at a temperature of $-19^{\circ} \mathrm{C}$. Coronal sections were $40 \mu \mathrm{m}$ in thickness, and every fifth section was collected. The recovered sections were stained with cresyl violet and dried at room temperature. Damage was quantified in the following way: For each subject, the AP coordinates of the recovered sections were identified using the Paxinos and Watson (2005) atlas. For each identified section, the intact hippocampus in each hemisphere was outlined using ImageJ (version 1.4; National Institutes of Health, Bethesda, Maryland). The areas of the hippocampus in both hemispheres were quantified (in pixels), and the total hippocampal area was estimated for each subject. Subsequently, the mean total hippocampal area in the shamlesioned group was calculated (one mean for each experiment), and the extent of hippocampal 
damage of each subject in the DHPC-lesioned group was estimated relative to the mean hippocampal area in the corresponding sham-lesioned group.

\title{
Data Treatment and Analyses
}

\author{
Measures of Timing Performance
}

For each subject, magazine entries in each 1-s bin of the non-reinforced peak trials were pooled across trials and sessions, resulting in a single conditioned-response timing distribution. Each timing distribution was smoothed over four 1-s bins. A Gaussian model with three parameters was then fitted onto each timing distribution,

$R_{i}=A e^{-\frac{1}{2}\left(\frac{x_{i}-C}{B}\right)^{2}}$

where $R_{i}$ indicates the conditioned-response rate in each 1-s bin. Parameter $A$, the peak conditioned-response rate (peak rate), indicates the strength of appetitive conditioning. $B$, the spread of the response distribution, reflects timing precision; greater spreads indicate less precise timing. $C$, the central tendency of the distribution, refers to the time point at which peak conditioned responding occurred (peak time). Based on these measures, we computed absolute error and coefficient of variation (cv)-absolute error was calculated as peak time - target time|, and coefficient of variation ( $c v)$ was calculated as spread / peak time; these are summarized in Figure 1. More absolute error indicates less accurate timing or higher timing variability within a group, while $c v$ indicates whether the spread of responding is proportional to the interval timed. If conditioned-response timing is timescale invariant, $c v$ should be approximately constant across studies or between groups. These five measures of timing (peak 
rate, spread, peak time, absolute error, and $c v$ ) were subject to analyses of variance (ANOVAs) in order to examine effects of DHPC lesion, stimulus duration, and modality.

Generalized Linear Mixed-Effects Models: Data Screening

To examine if there was any relationship between the extent of DHPC damage and different timing measures, a series of GLMMs was performed in R (R Core Team, 2013) with package lme 4 (version 1.0-6; Bates et al., 2014). Prior to the analyses, we screened for multicollinearity by examining partial correlations between different pairs of the variables to be included in the analyses. Bonferroni correction was applied, with $\alpha=.05 / n=.005$, where $n$ is the total number of comparisons to be made. We found that peak time was negatively correlated with $c v$ : controlling for peak rate, spread, absolute error, and CS duration; $r=-.755, p<.005$. In addition, the positive correlation between spread and $c v$ was almost significant: controlling for peak rate, peak time, absolute error, and CS duration; $r=+.527, p=.006$. These correlations are not surprising, given that $c v$ was computed from spread / peak time, and it implies that $c v$ is a redundant predictor in the presence of spread and peak time. No other correlations were significant after Bonferroni correction (Figure 2).

A more formal test for multicollinearity was performed by examining the variance inflation factors (VIFs) of the five predictors, peak rate, spread, peak time, absolute error, and $c v$. For each predictor $k$, a VIF was computed by $1 /\left(1-R^{2} k\right)$, where $R^{2} k$ represents the coefficient of determination obtained when $k$ was regressed against all other predictors. Only predictor $c v$ had a VIF > 10, which indicates serious multicollinearity. The remaining predictors had a mean VIF of 3.74 (range: 1.60-7.36). When predictor $c v$ was removed, VIFs of the remaining 
predictors decreased to a mean of 1.50 (range: 1.20-1.73), further confirming that $c v$ is a redundant predictor.

Generalized Linear Mixed-Effects Models: Defining Models and Model Selection

Based on the screening results, predictor $c v$ was removed from all further GLMM analyses. We started with a maximal model with peak rate, spread, peak time, and absolute error as fixed-effects predictors, and the extent of DHPC damage as the variable to be predicted. In terms of lme 4 syntax, the model was defined as:

$$
\begin{aligned}
& \text { damage } \sim \text { peak_rate }+ \text { spread }+ \text { peak_time + absolute_error }+ \\
& (1 \text { experiment })+(0+\text { cSlexperiment })
\end{aligned}
$$

This model consists of fixed-and random-effects components. The first four terms in the model were fixed effects: A slope with a fixed value $(\beta)$ was estimated for each of the four timing measures as in standard linear regression; $\beta$ coefficients that are greater than zero indicate positive relationships with the extent of DHPC damage, and values less than zero indicate negative relationships. The last two terms in the model were random effects:

(1 lexperiment) represents a random intercept for factor experiment $(1,2$, or 3$)$; and ( $0+$ CS I experiment) represents a random slope for factor CS duration (15 or $40 \mathrm{~s}$ ) or modality (visual or auditory), with factor experiment nested within it. The random component of the model takes into account, among other things, the different extent of DHPC damage across studies (see Histology results below).

We first assumed that the relationship between DHPC damage and any predictor to be linear, by adopting an identity link function $($ damage $=X \beta)$ and a normally distributed error structure in the dataset. We then explored the possibility that there might be nonlinear monotonic 
relationships, by adopting nonlinear link functions [hyperbolic: damage $^{-1}=X \beta$, or logarithmic: $\log ($ damage $)=X \beta]$ and a gamma error distribution in the dataset. But as the results (and subsequent discussion of the results) were similar under linear and nonlinear conditions, only the first set of GLMM finding (linearity assumed) was reported in the main article. The GLMM results obtained when nonlinearity was assumed can be found in supplementary Table S1.

To assess the importance of each timing measure in the maximal model, we adopted the model simplification approach (Crawley, 2007). A series of likelihood ratio tests was conducted, and each test compared a model with a particular predictor $(k)$ included against a model without that predictor. If the deletion of $k$ had no significant effect (as indicated by the $p$ value associated with the $\chi^{2}$ statistic), the model without $k$ was preferred (i.e. the more parsimonious model was retained). Predictor $k$ was retained in the model if $p<.05$.

Each of the four fixed-effects predictors was tested in turn, progressively reducing the full model to a minimal adequate model (Crawley, 2007). The order of factor deletion across steps was determined by how significant a factor was in predicting damage, as indicated by the $t$ value associated with each $\beta$ coefficient (the factor with the lowest absolute $t$ value was deleted first).

We also examined how well the actual values of DHPC damage and the predicted values from the best mixed-effects models were correlated. A strong correlation between the actual and predicted values would suggest that the amount of hippocampal damage could be predicted based on the linear combination of different timing measures. 


\section{Results}

\section{Histology}

Five subjects in the DHPC-lesioned group in Experiment 1 were excluded from further analyses because their hippocampal damage was mostly unilateral (Tam et al., 2013); the remaining 30 lesioned subjects all sustained bilateral damage to the anterior dorsal portions of the hippocampus; there was no damage to the dorsal subiculum. Hippocampal damage tended to start at AP bregma -1.80 mm (Plate 48; Paxinos \& Watson 2005) and extend to AP $-4.68 \mathrm{~mm}$ (Plate 72). The mean damage of the DHPC-lesioned subjects relative to total hippocampal volume of the sham-lesioned subjects in Experiments 1, 2, and 3 was $19.83 \%(n=7$, standard error $=1.17 \%), 33.65 \%(n=11$, standard error $=1.86 \%)$, and $37.98 \%(n=12$, standard error $=$ $2.42 \%$ ), respectively. ANOVA revealed that the mean hippocampal damage differed among the three experiments $[F(1,27)=16.648, p<.001]$, and post hoc Bonferroni tests indicated that there was less damage in Experiment 1 (15-s visual CS) than in Experiments 2 (15-s auditory CS) and 3 (40-s auditory CS), $p s<.005$; damage in Experiments 2 and 3 did not differ, $p=.401$. The relatively low percentage of damage in Experiment 1 is unlikely to be due to the coordinates used, which were the same as those in Experiment 3, and so must be attributable to some other factor (such as diffusion of neurotoxin).

One subject in the sham-lesioned group in Experiment 1 was excluded from analyses because some of its coronal sections were lost during the staining process and hence its dorsal hippocampal area could not be determined (Tam et al., 2013). The remaining 35 sham-lesioned subjects did not have any damage to the hippocampus or dorsal subiculum. Example 
photomicrographs from the sham- and DHPC-lesioned groups (15\% and 35\% damage) are shown in Figure 3A.

\section{Dorsal-Hippocampal Lesion Effects on Conditioned-Response Timing: Main Analyses}

Figures 3B-F show the group mean values of the five behavioural measures (peak rate, spread, peak time, absolute error, and $c v$ ) pooled across the three experiments; Table 2 shows the means in each experiment. Multiple Lesion $\times$ Experiment between-subjects ANOVAs revealed a significant main effect of Lesion on peak time as predicted, $[F(1,59)=6.629, p<.05$; Figure 3D], and a significant lesion effect was also found on $c v[F(1,59)=10.897, p<.005$; Figure $3 \mathrm{~F}]$. There was no Lesion $\times$ Experiment interaction for either peak time or $c v(p s>.7)$, suggesting that the lesion effect was similar across the three studies. No main effect of Lesion or Lesion $\times$ Experiment interaction was found for peak rate, spread, or absolute error (Figures 3B, 3C and 3E; $p s>.25)$. These results are summarized in Table 3. In addition, there were main effects of Experiment on peak rate, peak time and spread $(p s<.001)$, but not on error or $c v(p s>$ .2), effects which are likely to have resulted from the difference in CS duration among the three studies; this was examined further below.

Group mean response distributions during timing of 15-s (Experiments 1 and 2 combined) and 40-s target CSs (Experiment 3) on the non-reinforced peak trials are shown in Figures $4 \mathrm{~A}$ and $4 \mathrm{~B}$. When the target CS was $15-\mathrm{s}$, the mean response distribution of the DHPClesioned group was higher and broader than that of the sham-lesioned group (Figure 4A); when the target was $40 \mathrm{~s}$, there was no obvious difference between the group mean response distributions (Figure 4B).

\section{Effect of Lesion as a Function of CS Duration and Modality: Supplementary Analyses}




\section{(I) CS Duration}

We also took the opportunity to examine the effect of lesion as a function of CS duration across the three experiments. The group mean response distributions during timing of $15-\mathrm{s}$ (Experiments 1 and 2 combined) and 40-s target CSs (Experiment 3) were plotted on an absolute time scale in Figures 4C and 4D; in both groups these functions appeared higher and narrower for the 15-s than for the 40-s CS. When plotted on a relative time scale (Figures 4E and 4F) the mean response distributions for 15-s and 40-s targets superimposed, as would be predicted by Weber's law. The degree of overlap of the 15-s and 40-s timing distributions can be quantified in terms of $\eta^{2}$-a superposition index (range from $0-1$ ) that reflects the proportion of variance accounted for by the mean of the two distributions, with $\eta^{2}>.8$ indicating relatively good superposition in the literature (e.g., Brown et al., 1992). The values of $\eta^{2}$ in the sham- and DHPC-lesioned groups were relatively high and almost identical, .84 and .83 , respectively, suggesting that conditioned-response timing was timescale-invariant in the DHPC- as well as sham-lesioned groups.

To further examine this issue, group mean values for the different timing measures were computed as a function of CS duration (Figure 5), and CS Duration (15 s: Experiments 1 and 2 combined vs. $40 \mathrm{~s}$ : Experiment 3) $\times$ Lesion ANOVAs were conducted. In accordance with what was observed in Figures 4C and 4D, longer CSs yielded lower peak rate $[F(1,61)=10.160, p<$ $.005]$, but greater spread $[F(1,61)=606.996, p<.0005]$ and peak time $[F(1,61)=174.492, p<$ .0005]; there was no interaction between CS Duration and Lesion ( $p$ s >.3), suggesting that the degree of temporal discrimination (15 vs. $40 \mathrm{~s}$ ) was similar in the sham- and DHPC-lesioned groups (Figures 5A-C and 5F-H). No main effect of CS Duration or CS Duration $\times$ Lesion interaction was found with absolute error or $c v$ (all $p \mathrm{~s}>.14$; Figures 5D-E and 5I-J). The 
absence of an effect of CS Duration on $c v$ (spread / peak time) is particularly important, because it is consistent with the relatively good superposition of timing distributions within both groups, and confirms that DHPC lesion did not affect the timescale-invariance property of conditionedresponse timing. (As in the main analyses above, a main effect of Lesion was found for both peak time and $c v, p s<.02$, but not for other measures, $p \mathrm{~s}>.16$.)

\section{(II) CS Modality}

We also examined the difference between the lesioned and control groups as a function of CS modality by comparing the results from Experiments 1 and 2, which employed 15-s visual and auditory CSs respectively. Group mean response distributions for these studies, plotted on a relative time scale, are shown in Figures $4 \mathrm{G}$ and $4 \mathrm{H}$. The response distributions of the visual and auditory CSs superimposed relatively well during the early and middle portions of the nonreinforced test trial (from relative time 0-2), but not in the later portion of the test trial (shaded regions in Figures $4 \mathrm{G}$ and $4 \mathrm{H}$ ). In addition, the distributions of the auditory CS appeared to be broader than those for the visual CS, in both sham- and DHPC-lesioned groups. The values of $\eta^{2}$ in the sham- and DHPC-lesioned groups were almost identical, .64 and .63, respectively.

Group mean values for the five behavioural measures were computed as a function of CS modality for these two experiments; the resulting data are shown in Figure 6. Multiple CS Modality (visual: Experiment $1 v s$. auditory: Experiment 2) $\times$ Lesion ANOVAs revealed that auditory CSs yielded higher values of peak rate [Figures 6A and 6F; $F(1,37)=9.085, p<.01$ ] and absolute error [Figures 6D and 6I; $F(1,37)=5.459, p<.05$ ]; there was no interaction with Lesion for either measure ( $p s>.15$ ), suggesting that the effect of CS Modality was similar in the sham- and DHPC-lesioned groups. No main effect of CS Modality or CS Modality $\times$ Lesion 
interaction was found with spread (Figures 6B and 6G), peak time (Figures 6C and 6H), or $c v$ (Figures 6E and 6J), all $p s>.15$. (As in the main analysis above, a main effect of Lesion was found for both peak time and $c v, p s<.003$, but not for other measures, $p \mathrm{~s}<.4$.)

\section{Discussion}

The analysis of peak time pooled over the three experiments confirmed that the DHPClesioned subjects underestimated target durations of $15 \mathrm{~s}$ and $40 \mathrm{~s}$, consistent with our previous findings (Tam \& Bonardi, 2012a; Tam et al., 2013; see also Jaldow et al., 1989; Balci et al., 2009; Yin \& Meck, 2014), and the size of this DHPC-lesion effect on timing accuracy did not differ across studies (Tam \& Bonardi, 2012a,b; Tam et al., 2013). Within each group, conditioned-response timing was timescale invariant, as indicated by relatively good superposition of 15-s and 40-s timing distributions plotted on a relative time scale, as well as the non-significant effect of CS Duration on $c v$. However, the DHPC-lesioned group had a higher mean $c v$ than the sham-lesioned subjects. This implies that DHPC damage produced a nonscalar increase in timing variability. But this increase in timing variability in the DHPC-lesioned subjects was not accompanied by greater absolute error in these animals (cf. Tam \& Bonardi, 2012b).

Peak rate and absolute error were higher when timing a 15-s auditory CS than when timing a visual CS of the same duration, although there was no difference in spread, peak time, or $c v$. The sensory modality effects on peak rate and absolute error were, however, unaffected by DHPC lesion. In accordance with these findings, the visual and auditory CS timing distributions did not superimpose well in either group when plotted on relative scales, with the auditory CS timing distributions broader and noisier (i.e. less smooth) than the visual CS 
distributions. However, it should be emphasised that there were other differences in procedure between the three experiments in addition to the differences in sensory modality and CS duration considered above, such as inter-trial interval, total number of conditioning trials, and total number of non-reinforced test trials. Thus it is necessary to be cautious in attributing the differences outlined above to CS modality or duration.

\section{Relationship between Extent of Dorsal Hippocampal Damage and Measures of Conditioned-response Timing}

In all three studies there was variability both in the extent of DHPC damage and in the five different measures of behavior. Data of this type provide an opportunity to examine the relationship between the extent of DHPC damage and timing performance in a more quantitative manner. Figures 7A-E show the scatter plots of the extent of DHPC damage $v s$. different aspects of timing.

In order to take into account the differential amount of DHPC damage and parametric differences across studies, we conducted a series of GLMMs with factor experiment, CS duration (15 or $40 \mathrm{~s}$ ), and modality (visual or auditory) as random effects. The different measures, peak rate, spread, peak time, and absolute error, were entered as fixed-effects predictors; $c v$ was not included based on the results from multicollinearity screening. The extent of DHPC damage was entered as the variable to be predicted by the above fixed- and random-effects factors. The lme 4 syntax of the different GLMMs is summarized in Table 4.

Among the four fixed-effects predictors in the maximal model, peak time was the weakest predictor of damage $(\beta=.195$, standard error $=.159, t=1.224)$. No significant effect 
was found when peak time was deleted from the model $\left(\chi^{2}=1.365, p=.243\right)$. The removal of peak time resulted in a simpler model in which spread was the weakest predictor of damage $(\beta=$ -.244 , standard error $=.167, t=-1.461)$. No significant effect was found when spread was deleted from the model $\left(\chi^{2}=1.581, p=.209\right)$.

This resulted in a model with only two fixed-effects predictors, which represents our minimal adequate model, as significant effects were obtained when peak rate was deleted $\left(\chi^{2}=\right.$ 7.336, $p<.01)$, and when absolute error was deleted $\left(\chi^{2}=5.062, p<.05\right)$. Furthermore, reference to Table 4 shows that these two models (steps $4 a$ and $4 b$ ) were more than 2 AIC (Akaike's information criterion) values greater than the minimal adequate model with peak rate and absolute error included (step 3 in Table 4); thus, the model with these two predictors represents the best model in the set (Burnham \& Anderson, 2002; Crawley, 2007). Thus, in addition to the contribution of the random component in the model (factor experiment, $C S$ duration, or modality), the extent of DHPC damage was positively predicted by peak rate $(\beta=$ .275$, standard error $=.089, t=3.099)$ and by absolute error $(\beta=.461$, standard error $=.196, t=$ 2.358). We checked if there was any interactive effect between the two fixed-effects predictors, peak rate $\times$ absolute error, but no significant effect was found when the interaction terms were added to the minimal adequate model $\left(\chi^{2}=.002, p=.967\right)$.

We then examined how well the actual DHPC damage values could be predicted from the minimal adequate model with peak rate and absolute error as fixed-effects predictors. The correlation between the actual and predicted values of damage was strong: $r=+.813, p<.0001$ (Figure 8A). Importantly, the correlation was of the same magnitude as the correlation between the predicted values from the maximal model and actual DHPC damage: $r=+.837, p<.0001$ (Figure 8B). Therefore, the severity of DHPC damage was predicted based on the linear 
combination of peak rate and absolute error. In addition we employed a more conservative jackknife approach (Crawley, 2007), to examine how well $\beta$ estimates from a smaller subsample of rats could be used to predict DHPC damage in rats not included in the model. More specifically, each animal was left out from the dataset, one at a time, and $\beta$ estimates for peak rate and absolute error were recalculated based on the smaller sample $(n=30-1)$. The new set of $\beta$ estimates was then used to predict the extent of DHPC damage of the animal not included in the model; this procedure was repeated for each of the 30 DHPC-lesioned animals. The correlation between the actual and predicted values of damage based on this jackknife approach remained strong: $r=+.702, p<.0001$, demonstrating cross validity of the minimal adequate model. Frequency distributions of the $\beta$ estimates for peak rate and absolute error obtained from the jackknife method are shown in Figures 9A and 9B, respectively.

Finally, we examined if the significant relationships between damage and peak rate and absolute error differed for different stimulus duration or modality, by testing for any significant damage $\times$ CS duration or damage $\times$ modality interactive effect on the two measures:

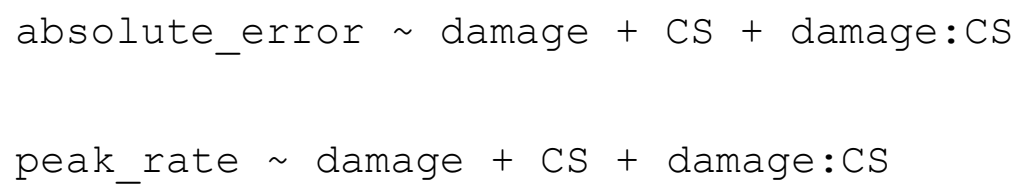

The last terms in the above models represent the interactions of interest. With respect to absolute error, there was no interaction between damage and CS duration $(\beta=.309$, standard error $=$ $.267, t=1.161, p=.256)$, or between damage and modality $(\beta=.655$, standard error $=.801, t=$ $.818, p=.421)$.

With respect to peak rate, there was no damage $\times$ modality interaction $(\beta=-3.350$, standard error $=2.172, t=-1.543, p=.135)$. However, the damage $\times C S$ duration interaction 
was almost significant $(\beta=-1.078$, standard error $=.528, t=-2.040, p=.052)$, implying that the relationship between damage and peak rate might be different for 15 -s and 40-s CSs. Consistent with this idea, when only the 15-s CS data were analysed, there was a positive relationship between damage and peak rate $(\beta=1.313$, standard error $=.359, t=3.653, p<.005)$, but no significant relationship was evident when the 40-s CS data were analysed $(\beta=.235$, standard error $=.337, t=.696, p=.502)$.

\section{Discussion}

The extent of DHPC damage was significantly related to, and could be well predicted by, peak rate and absolute error (Tables 3 and 4), both of which increased with lesion extent. For absolute error, the strength of the positive relationship appeared to be similar for 15-s vs. 40-s CSs and for stimulus of different modality, but for peak rate, the positive relationship was more evident when the CS was $15 \mathrm{~s}$ than when it was $40 \mathrm{~s}$ (although this latter interactive effect only approached significance).

\section{General Discussion}

A comparison of performance in the DHPC- and sham-lesioned subjects showed that DHPC-lesioned subjects display maximal responding at significantly earlier time points than the sham-lesioned subjects (Figure 3D), suggesting that they tended to underestimate the duration of the CS. This is consistent with previous reports on the effects of fimbria-fornix lesions (e.g., Meck et al., 1984; Meck, 1988), and confirms that damage to the DHPC is sufficient to produce 
these effects. However, the lower peak time in the lesioned animals was not accompanied by the corresponding decrease in spread that Weber's law would predict (Figure 3C), with the result that $c v$ was significantly larger in the DHPC-lesioned than control subjects (Figure 3F). This indicates that, in addition to an impairment in accuracy, there is also a reduction in timing precision after DHPC damage - an effect that has not previously been reported. To our knowledge only two studies have examined this, and both reported that the reduction in peak time produced by hippocampal damage was accompanied by a reduction in spread (Meck et al., 1984; Buhusi \& Meck, 2002; see MacDonald 2014 for a recent review) - in complete contrast to the results reported here. However, these prior studies employed lesions of the fimbria-fornix (Meck et al., 1984) and ibotenic acid lesions of the entire hippocampus (Buhusi \& Meck, 2002). It is possible that the difference in lesion methods could account for the contrast between their findings and those of the studies reported here. Finally, and also consistent with most previous reports, was the observation that these effects of dorsal hippocampal lesion on appetitive conditioned-response timing were selective-they did not impair appetitive conditioning (Figure $3 B)$.

The results from the GLMMs complemented these findings, by examining the extent to which the different variables in combination were able to predict the degree of damage that was present; because all the variables were represented in the same model, it was possible to determine which of them were most influential, while at the same time controlling for parametric variations among the three studies. We are not aware of any other studies that have examined the relationship between degree of hippocampal damage and timing parameters. Our analysis revealed that damage was not strongly predicted by peak time (Figure 7C), but that there was a strong relationship between damage and absolute error (Figure 7D), a measure which is blind to 
whether animals under- or over-estimated the target duration (cf. Tam \& Bonardi, 2012b). A second finding from GLMMs was the positive relationship between the extent of DHPC damage and appetitive conditioned responding (Figure 7A). This result is at face value surprising: Although peak rate was numerically higher in the lesioned than in the control subjects in Experiments 1 and 2, this difference was not significant in ANOVA, and there have been almost no previous reports of DHPC lesion influencing delay conditioned responding. An exception is Tam and Bonardi (2012b) who found the opposite effect-that DHPC lesions impaired conditioned responding during initial conditioning sessions with the 40-s CS (see also Beylin et al., 2001, who reported a parallel impairment with longer CSs in a delay eyeblink conditioning preparation).

The present results confirm and extend existing evidence that the DHPC is important for temporal processing (Olton, 1986; Kesner, 1998; Sakata, 2006; Howard \& Eichenbaum, 2013). First, we replicated previous reports that hippocampal dysfunction reduces peak time, and confirm that damage to the dorsal portion of this structure is sufficient to produce these effects. They also demonstrated a previously unreported effect, that damage to the DHPC also appears to reduce the precision of timing: $c v$ was greater in the lesioned than in control subjects, which implies the scalar property of timing was violated such that timing precision did not decrease as the observed peak time decreased. Moreover, the GLMM analysis suggested that there was a strong predictive relation between absolute timing error and amount of DHPC damage. Both findings suggest greater variability in interval timing after DHPC damage, and the effect on $c v$ suggests that this is independent of the duration being timed. The implication is that DHPC damage might have multiple effects on the timing process. 
The effects of hippocampal damage on interval timing are usually interpreted in terms of scalar timing theory (Gibbon et al., 1984). According to this model, timing behavior requires of a pacemaker which emits pulses that are transferred to an accumulator via a switch when a stimulus is presented. When a US is encountered, the total number of pulses emitted between the stimulus onset and US delivery is then transferred into a long-term memory store, multiplied by a constant $K$ that is close, but not equal, to one. When the same stimulus is re-encountered, the number of accumulating pulses in the accumulator is compared with one of the values stored in long-term memory, and conditioned responding begins when these values are sufficiently close. In terms of this model, the behavioral effects of hippocampal, and more specifically DHPC damage, are typically interpreted as a reduction in $K$. This results in the values stored in longterm memory being systematically shorter than those transferred from the accumulator, resulting in animals responding too early (e.g., Meck et al., 2013). However, if a change in $K$ were the only effect of DHPC damage, scalar invariance should be unaffected: A reduction in $K$ should produce a reduction in peak time, but also a corresponding reduction in spread, with the result that $\mathrm{cv}$ should be constant—and as we saw above, this was not the case. The finding that $\mathrm{cv}$ was greater in the DHPC-lesioned animals, and that absolute error in timing was systematically related to degree of DHPC damage, could be taken to suggest that DHPC damage does more than just reduce $K$.

One possibility, for example, is that it changes the criterion used to determine whether the current value in the accumulator is sufficiently close to the value taken from long term memory to produce a response. If DHPC lesion results in a constant value being added to the discrepancy between the current timed interval and the stored value that is required for responding to occur, the lesioned subjects would start responding at earlier time points and cease responding at later 
ones, leading to an increase in spread (i.e. less precise timing) that is independent of the duration the timed event. The same result would be observed if a constant value were added to the time taken to open the switch that controls transfer of pulses from the pacemaker to the accumulator. Either of these effects could produce the pattern of results that we observed.

A second, and somewhat surprising finding, was that of the positive relationship between asymptotic rate of conditioned responding and DHPC damage. The first reason this is surprising is because, although an impairment on trace conditioning is regarded by some as diagnostic of hippocampal damage, HPC lesions typically have little effect on the rate of conditioned responding in delay conditioning tasks. However, as mentioned above, there have been a few reports of CR disruption in studies in which longer CSs are employed (Beylin et al., 2001; Tam \& Bonardi, 2012b). As longer CSs, like trace-conditioned CSs, tend to support relatively low rates of conditioned responding, it may be that the failure of most studies to observe an effect is simply due to lack of sensitivity in the conditioning measure. But the second surprising aspect is the direction of the relationship — the greater the damage the higher the rate of conditioned responding. However, relationships of this type are not without precedent. For example, Baxter and Murray (2001) reported a positive relationship between the magnitude of hippocampal damage and performance on a visual trial-unique delayed non-matching-to-sample task in macaques; performance on this task is typically worse in subjects with hippocampal damage than control animals (although see Zola \& Squire, 2001). They suggested three possible mechanisms that might underlie such an observation. First, partial damage to the hippocampus could also alter activity in adjacent structures that are responsible for a particular behavioral output which might enhance performance, while more complete hippocampal damage would minimize such an effect. Second, they suggested that the hippocampus might compete with some other structure to 
achieve a behavioral goal—such that increasing HPC damage would increasingly release the second structure from this competition, thus enhancing performance. Finally, they proposed that less HPC damage might be accompanied by more extra-HPC damage caused by misdirected neurotoxin. We think this latter suggestion unlikely in the present studies, in which the lesions were small and confined to the DHPC; but the first two are viable possibilities, and could even explain why DHPC lesions have been reported to have a disinhibitory effect on appetitive responding (Cheung \& Cardinal 2005).

In principle it is possible that the effects of DHPC damage on peak response rates, spreads, and absolute timing errors could be related. For example, a general disinhibition of conditioned responding that increases with DHPC damage could effectively make the criterion postulated by scalar timing theory less conservative: Animals would start responding earlier and stop responding later in time on each trial, thereby increasing the spread of the overall response distribution (and probably absolute timing error) when data are combined across multiple trials. Thus, a significant correlation between peak rate and spread, or between peak rate and absolute error in timing, would be expected if these measures are interrelated. There was, however, no statistically significant correlation between pairs of these three variables; correlation coefficients are shown in the heatmap in Figure 2. These negative results do not provide evidence for the idea that the increased timing error was secondary to an increase in peak response rate.

These behavioral findings complement the increasing body of evidence from single-unit recording studies, that cells in the DHPC show firing patterns that are dependent on the temporal structure of the learning episode—not only anticipating the time of delivery of both signalled (McEchron et al., 2003) and unsignalled USs (Delacour \& Houcine, 1987; Young \& McNaughton, 2000), but also showing populations of cells that seem to track both absolute and 
relative time (MacDonald et al., 2011; Naya \& Suzuki, 2011). However, the question remains as to whether the DHPC's role in timing is a primary or secondary one.

A recent, extremely influential model of timing is the Striatal Beat Frequency model (SBF; Matell \& Meck, 2000, 2004; Buhusi \& Oprisan, 2013). This model, which is one of the first that attempts to translate theoretical timing principles into neurobiological substrates, asserts that striatal medium spiny neurons (MSNs) detect the temporal coincidence between reinforcement and activity in a subset of the cortical neurons from which they receive input. The different intrinsic frequencies of these cortical neurons allow them to oscillate at different periodicities, and by detecting such patterns the MSNs can be trained to respond to different durations. According to this view the role of the hippocampus in timing is a secondary one, perhaps through its tonic inhibitory effect on the striatum (Meck, 1988; Meck et al., 2013). Moreover, Yin and Troger (2011) recently suggested a number of ways in which the hippocampus could interact with this cortico-striatal timing network. They suggested that the hippocampus might modulate the memory stage of the timing process, which could be responsible for the typically observed leftward shift in peak stage. But interestingly they also proposed two further mechanisms that could underlie our demonstration that DHPC lesions increased timing variability. First, they suggested that the hippocampus might regulate the dynamic firing thresholds of the striatal MSNs, which they argued could produce more trial-by trial noise in timing performance. Second they suggested that it might affect the decision process that controls responding — arguably equivalent to the response threshold in scalar timing theory. Variation in this threshold could also underlie the greater error in timing that we found here.

A contrasting approach to this issue has recently been suggested by MacDonald (2014). His argument is that there is an inconsistency between the idea that the hippocampus is 
secondary to cortico-striatal networks in mediating timing, and its well-established role in encoding the temporal organisation of events in episodic memory-type tasks. He proposes that the effective relegation of the hippocampus to a supporting role in timing may stem from the fact that most neural and behavioural investigations of hippocampal involvement in timing have relied on prospective memory paradigms, in which memory for a specific duration is required by the structure of the task. The peak procedure is an example of this, as animals will be motivated to learn the point at which food is delivered. In contrast, in retrospective paradigms learning about duration is incidental to the task, and only probed afterwards. He argues that retrospective procedures are more likely to tap the type of temporal encoding that episodic-type tasks require, and thus to be those in which the hippocampus is critical. These various parallels are all intriguing; nonetheless, further work will be required to clarify the exact nature of the hippocampus's contribution to timing processes (see Bonardi et al., 2014). 


\section{References}

Aggleton JP, Poirier GL, Aggleton HS, Vann SD, Pearce JM. 2009. Lesions of the fornix and anterior thalamic nuclei dissociate different aspects of hippocampal-dependent spatial learning: Implications for the neural basis of scene learning. Behav Neurosci 123:504-519.

Balci F, Meck WH, Moore H, Brunner D. 2009. Timing deficits in aging and neuropathology. In: Bizon JL, Woods A, editors. Animal models of human cognitive aging. Totowa, NJ: Humana Press. p $1-41$.

Bates D, Maechler M, Bolker B, Walker S. 2014. Packages 'Ime4': Linear mixed-effects models using Eigen and S4. http://cran.r-project.org/web/packages/lme4/lme4.pdf

Baxter MG, Murray EA. 2001. Opposite relationship of hippocampal and rhinal cortex damage to delayed nonmmatching-to-sample deficits in monkeys. Hippocampus 11:61-71.

Beylin AV, Gandhi CC, Wood GE, Talk AC, Matzel LD, Shors TJ. 2001. The role of the hippocampus in trace conditioning: Temporal discontiguity or task difficulty? Neurobiol Learn Mem 76:447-461.

Bonardi C, Cheung THC, Mondragón E, Tam SKE. 2014. The temporal characteristics of associative learning and its neural substrates. In: Murphy R, Honey RC, editors. The WileyBlackwell handbook on the cognitive neuroscience of learning. Hoboken, NJ: Wiley-Blackwell.

Brown BL, Hemmes NS, De Vaca SC. 1992. Effects of intratrial stimulus change on fixedinterval performance: The roles of clock and memory processes. Anim Learn Behav 20:83-93. 
Buhusi CV, Meck WH. 2002. Ibotenic acid lesions of the hippocampus disrupt attentional control of interval timing. Program no. 183.10, Neuroscience meeting planner. Orlando, FL: Society for Neuroscience. Online.

Buhusi CV, Oprisan S. 2013. Time-scale invariance as an emergent property in a perceptron with realistic, noisy neurons. Behav Processes 95:60-70.

Burnham KP, Anderson DR. 2002. Avoiding pitfalls when using information-theoretic methods. $J$ Wildl Manag 66:912-918.

Burnham KP, Anderson DR, Huyvaert KP. 2011. AIC model selection and multimodel inference in behavioral ecology: Some background, observations, and comparisons. Behav Ecol Sociobol 65:23-25.

Cheung THC, Cardinal RN. 2005. Hippocampal lesions facilitate instrumental learning with delayed reinforcement but induce impulsive choice in rats. BMC Neuroscience 6:36.

Crawley MJ. 2007. The R book. Chichester, England: John Wiley \& Sons. 950 p.

Delacour J, Houcine O. 1987. Conditioning to time: Evidence for a role of hippocampus from unit recording. Neuroscience 23:87-94.

Field A, Miles J, Field Z. 2012. Discovering statistics using R. London: SAGE Publications Ltd. $992 \mathrm{p}$.

Gallistel CR, Gibbon J. 2000. Time, rate, and conditioning. Psychol Rev 107:289-344.

Gibbon J., Balsam P. 1981. Spreading association in time. In: Locurto LC, Terrace HS, Gibbon J, editors. Autoshaping and conditioning theory. New York: Academic Press. p 219-253. 
Gibbon J, Church RM, Meck WH. 1984. Scalar timing in memory. Ann N Y Acad Sci 423:52-77.

Howard MW, Eichenbaum H. 2013. The hippocampus, time, and memory across scales. J Exp Psychol Gen 142:1211-1230.

Jaldow EJ, Oakley DA, Davey GC. 1989. Performance of decorticated rats on fixed interval and fixed time schedules. Eur J Neurosci 1:461-470.

Jung MW, Wiener SI, McNaughton BL. 1994. Comparison of spatial firing characteristics of units in dorsal and ventral hippocampus of the rat. J Neurosci 14:7347-7356.

Kesner RP. 1998. Neural mediation of memory for time: Role of the hippocampus and medial prefrontal cortex. Psychon Bull Rev 5:585-596.

Kjelstrup KB, Solstad T, Brun VH, Hafting T, Leutgeb S, Witter MP, Moser EI, Moser MB. 2008. Finite scale of spatial representation in the hippocampus. Science 321:140-143.

MacDonald CJ, Lepage KQ, Eden UT, Eichenbaum H. 2011. Hippocampal “time cells" bridge the gap in memory for discontiguous events. Neuron 71:737-749.

MacDonald CJ. 2014. Prospective and retrospective duration memory in the hippocampus: is time in the foreground or background? Phil Trans R Soc B 369:20120463.

McEchron MD, Tseng W, Disterhoft JF. 2003. Single neurons in CA1 hippocampus encode trace interval duration during trace heart rate (fear) conditioning in rabbit. J Neurosci 23:1535-1547.

Matell MS, Meck WH. 2000. Neuropsychological mechanisms of interval timing behavior. Bioessays 22:94-103. 
Matell MS, Meck WH. 2004. Cortico-striatal circuits and interval timing: Coincidence detection of oscillatory processes. Cogn Brain Res 21:139-170.

Meck WH, Church RM, Olton DS. 1984. Hippocampus, time, and memory. Behav Neurosci 98:3-22.

Meck WH. 1988. Hippocampal function is required for feedback control of an internal clock's criterion. Behav Neurosci 102:54-60.

Meck WH, Church RM, Matell MS. 2013. Hippocampus, time, and memory: A retrospective analysis. Behav Neurosci 127:642-654.

Moser EI, Moser MB, Andersen P. 1993. Spatial learning impairments parallels the magnitude of dorsal hippocampal lesions but is hardly present following ventral lesions. J Neurosci 13:39163925.

Moser MB, Moser EI, Forrest E, Andersen P, Morris RGM. 1995. Spatial learning with a minislab in the dorsal hippocampus. Proc Natl Acad Sci U S A 92:9697-9701.

Naya Y, Suzuki WA. 2011. Integrating what and when across the primate medial temporal lobe. Science 333:773-776.

Olton DS. 1986. Hippocampal function and memory for temporal context. In: Isaacson RL, Pribram KH, editors. The hippocampus, volume 4. New York: Plenum Press. p 281-298.

Paxinos G, Watson C. 2005. The rat brain in stereotaxic coordinates, $5^{\text {th }}$ edition. New York: Elsevier Academic Press. 209 p. 
Penney TB, Gibbon J, Meck WH. 2000. Differential effects of auditory and visual signals on clock speed and temporal memory. J Exp Psychol Hum Percept Perform 26:1770-1787.

R Core Team. 2013. R: A language and environment for statistical computing. Vienna, Austria: R Foundation for Statistical Computing. http://www.R-project.org/

Royer S, Sirota A, Patel J, Buzsáki G. 2010. Distinct representations and theta dynamics in dorsal and ventral hippocampus. J Neurosci 30:1777-1787.

Sakata S. 2006. Timing and hippocampal theta in animals. Rev Neurosci 17:157-162.

Sanderson D, Pearce JM, Kyd R, Aggleton JP. 2006. The importance of the rat hippocampus for learning the structure of visual arrays. Eur J Neurosci 24:1781-1788.

Tam SKE, Bonardi C. 2012a. Dorsal hippocampal involvement in appetitive trace conditioning and interval timing. Behav Neurosci 126:258-269.

Tam SKE, Bonardi C. 2012b. Dorsal hippocampal lesions disrupt Pavlovian delay conditioning and conditioned-response timing. Behav Brain Res 230:259-267.

Tam SKE, Jennings DJ, Bonardi C. 2013. Dorsal hippocampal involvement in conditionedresponse timing and maintenance of temporal information in the absence of the CS. Exp Brain Res 227:547-559.

Yin B, Troger AB. 2011. Exploring the 4th dimension: Hippocampus time and memory revisited. Front Integr Neurosci 5:36. 
Yin B, Meck WH. 2014. Comparison of interval timing behaviour in mice following dorsal or ventral hippocampal lesions with mice having $\delta$-opioid receptor gene deletion. Phil Trans $R$ Soc B 372:20120466.

Young B, McNaughton N. 2000. Common firing patterns of hippocampal cells in a differential reinforcement of low rates of response schedule. J Neurosci 20:7043-7051.

Zola SM, Squire LS. 2001. Relationship between magnitude of damage to the hippocampus and impaired recognition memory in monkeys. Hippocampus 11:92-98. 


\section{Figure Captions}

Figure 1. Measures of conditioned-response timing. Five different measures of interval timing were obtained from fitting a Gaussian function (Fitted, black line) onto each individual's response distribution (Observed, grey line): 1. Peak rate is the maximum response rate (in responses per minute; rpm); 2. spread indicates the width of the response distribution (in seconds); and 3. peak time (in seconds) is the time point at which maximum responding occurred. From spread and peak time, we computed two other measures: 4. absolute timing error (in seconds), which is the absolute difference between peak time and target CS duration; and $\mathbf{5 .}$ coefficient of variation ( $c v)$ which is calculated from spread divided by peak time. If conditioned-response timing is timescale invariant, the mean $c v$ of each group should be approximately constant across studies with different stimulus duration and modality.

Figure 2. Absolute partial correlation values $(|r|)$ between different pairs of the five variables after DHPC lesion. The "hotter" the colour on the heatmap, the stronger was the relationship between two variables (while "partialling” out all other variables). * indicates correlation was almost significant after Bonferroni correction ( $p$ slightly above .005$)$; $\uparrow$ indicates correlation was statistically significant after Bonferroni correction $(p<.005)$.

Figure 3. Effects of DHPC lesion on conditioned-response timing performance. Panel

A, from left to right columns: Example coronal sections from a representative sham-lesioned subject, a DHPC-lesioned subject sustaining approximately $15 \%$ of hippocampal damage, and a DHPC-lesioned subject sustaining approximately $35 \%$ of hippocampal damage. The top, middle, and bottom rows show, respectively, coronal sections about $2.28 \mathrm{~mm}, 2.76 \mathrm{~mm}$, and $3.48 \mathrm{~mm}$ posterior to bregma, which correspond to plates 52, 56, and 62 in the Paxinos and Watson (2005) 
atlas. Panels B-F: Effects of DHPC lesion on peak rate, spread, peak time, absolute error, and $c v$. Asterisks $(*)$ indicate significant main effects of DHPC lesion (on peak time and $c v$ ) in between-subjects Lesion $\times$ Experiment ANOVAs. Error bars indicate standard error of the mean.

Figure 4. Group mean conditioned-response timing distributions. Panels A and B: The mean response distributions of the sham-lesioned group (grey lines) and DHPC-lesioned group (red lines) when the target CS was $15 \mathrm{~s}$ (visual and auditory CSs combined) and $40 \mathrm{~s}$ (auditory CS); vertical lines indicate target CS duration. The response distribution of the DHPC-lesioned group was higher and broader than that of the sham-lesioned group when the CS was $15 \mathrm{~s}$ but not when it was 40 s. Panels $\mathbf{C}$ and D: The 15-s and 40-s timing distributions of each group from panels $\mathbf{A}$ and $\mathbf{B}$ were plotted on the same graph. In both groups, the 15-s distributions (red lines) were higher and narrower than the 40-s response distributions (green lines; vertical lines indicate target CS duration). Panels $\mathbf{E}$ and $\mathbf{F}$ : The timing distributions from panels $\mathbf{C}$ and $\mathbf{D}$ superimposed when they were plotted on a relative time scale ( $t / \mathrm{CS}$ duration; 1 indicates target CS duration), suggesting that conditioned-response timing is timescale invariant. The degree of overlapping of the 15-s and 40-s distributions can be quantified in terms of $\eta^{2}$, a superposition index (ranged from 0-1) that reflects the proportion of variance accounted for by the mean of the two distributions. $\eta^{2}>.8$ indicates relatively good superposition in the literature (e.g., Brown et al., 1992). Panels G and H: For both the sham- and DHPC-lesioned groups, the 15-s timing distributions for the visual CS (red lines) and auditory CS (green lines) did not superimpose well when they were plotted on a relative time scale, especially beyond relative time 2 (indicated by the shaded regions. In general, the visual CS distributions were broader and less smooth than the auditory CS distributions, suggestive of a sensory modality effect on response timing. 
Figure 5. Effects of stimulus duration on conditioned-response timing. Panels A-E:

Effects of CS duration (15 vs. $40 \mathrm{~s}$ ) on different measures in the sham-lesioned subjects; panels F-J: Effects of CS duration in the DHPC-lesioned subjects. Asterisks (*) indicate significant main effects of CS duration (on peak rate, spread, and peak time) in CS Duration $\times$ Lesion ANOVAs. Error bars indicate standard error of the mean.

Figure 6. Effects of stimulus modality on conditioned-response timing. Panels A-E: Effects of CS modality (visual vs. auditory) on different measures in the sham-lesioned subjects; panels F-J: Effects of CS modality in the DHPC-lesioned subjects. Asterisks (*) indicate significant main effects of CS modality (on peak rate and absolute error) in CS Modality $\times$ Lesion ANOVAs. Error bars indicate standard error of the mean.

Figure 7. Extent of DHPC damage in the DHPC-lesioned group and timing

performance. Panels A-E: Scatter plots showing the relationships between different measures of conditioned-response timing and amount of hippocampal damage. The extent of DHPC damage was significantly related to peak rate (panel A) and absolute error (panel D). Data points from Experiment 1 (Tam et al., 2013) are marked in red; data points from Experiment 2 (Tam \& Bonardi, 2012a) are marked in green; and those from Experiment 3 (Tam \& Bonardi, 2012b) are marked in blue.

Figure 8. Actual vs. predicted hippocampal damage from mixed-effects models. Panel A: Predicted values of damage were obtained from the minimal adequate models with peak rate and absolute error as fixed-effects predictors (step 3 in Table 4); the correlation between actual damage (blue line) and predicted damage (red line) was +.813 . Panel B: Predicted values of damage were obtained from the maximal models with peak rate, spread, peak time, and absolute 
error as fixed-effects predictors (step 1 in Table 4); the correlation between actual damage (blue line) and predicted damage (red line) was +.837 , which is of the same magnitude as the correlation in panel $\mathbf{A}$.

Figure 9. Histograms showing mean $\beta$ coefficients and variance for the fixed-effects predictors in the minimal adequate model obtained from the jackknife method. Panels A and B: Frequency distributions (grey bars) of $\beta$ coefficients for peak rate and absolute error, respectively; smooth lines (red) represents probability density functions of normal distributions with mean $=.277$, standard deviation $=.026$ and mean $=.465$, standard deviation $=.056$, respectively. Mean $\beta$ coefficients were significantly different from the value of zero (null effect) in both panels according to one-sample $t$ tests ( $p<.005,2$-tailed), suggesting that peak rate and absolute error were related to the extent of DHPC damage. 
Figure 1.

Timing Measures 1-5

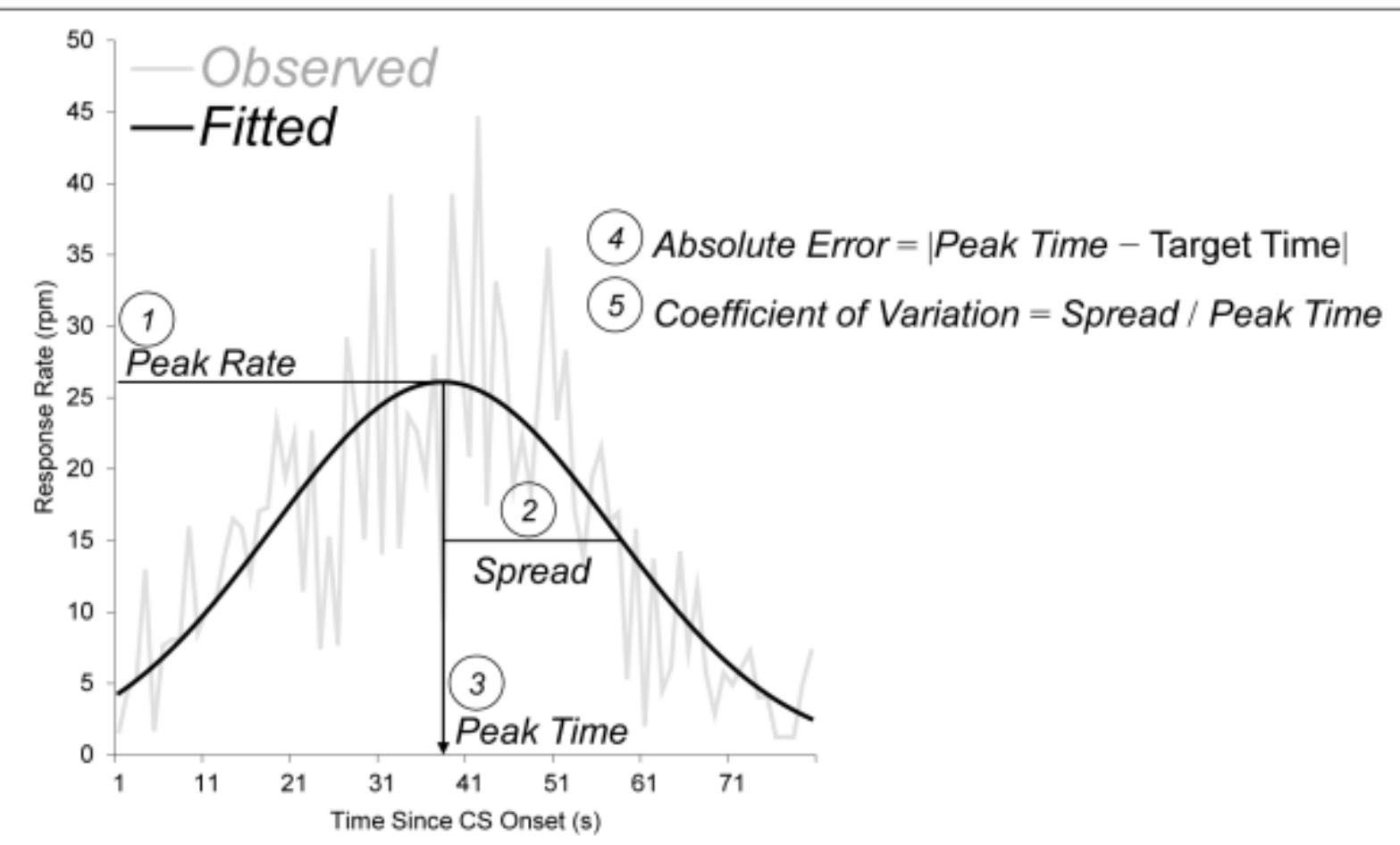


Figure 2.

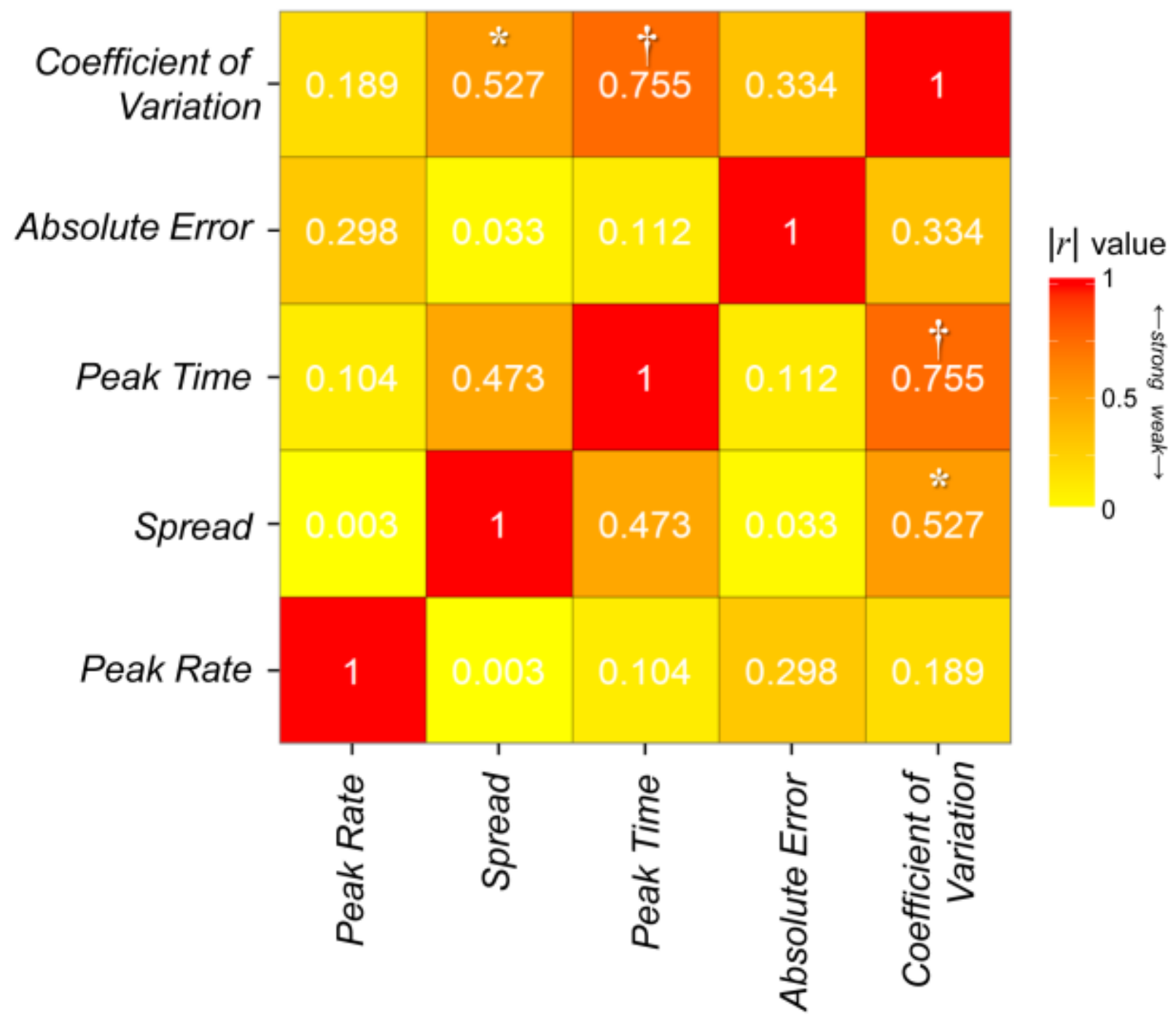


Figure 3.

\section{Lesion}

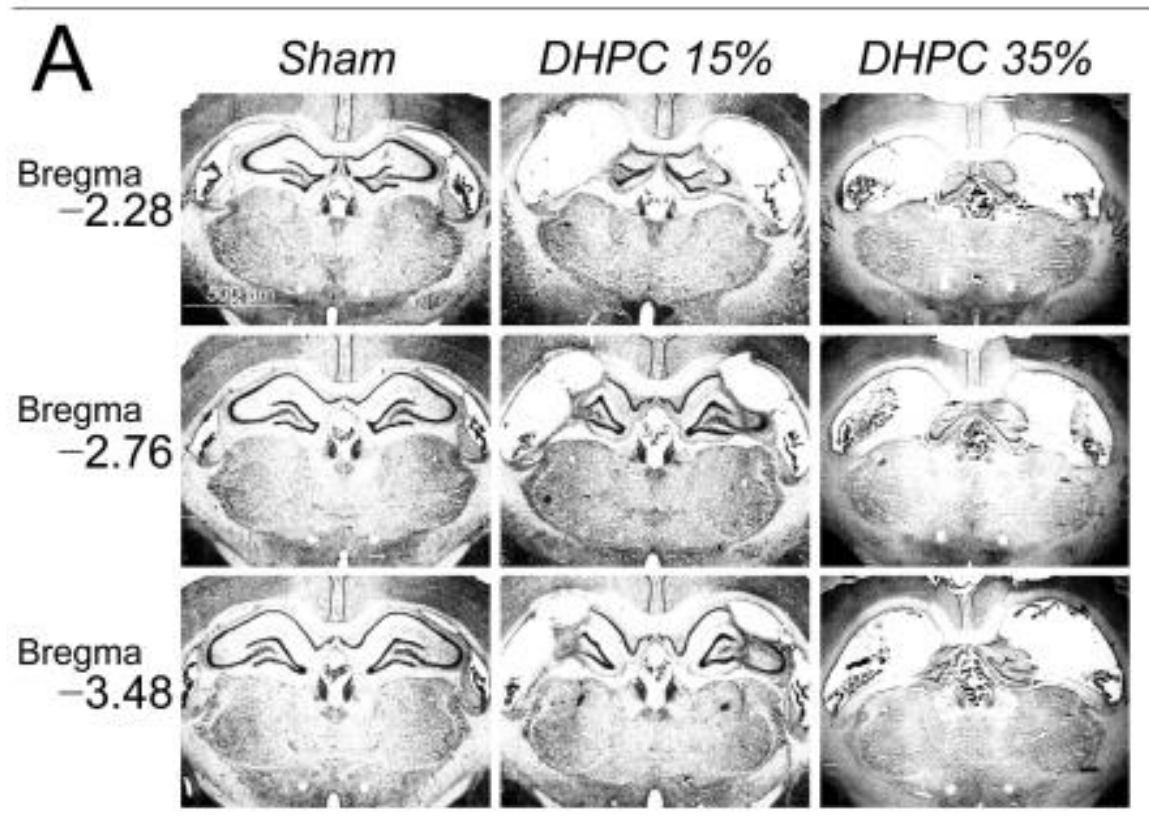

Timing Performance

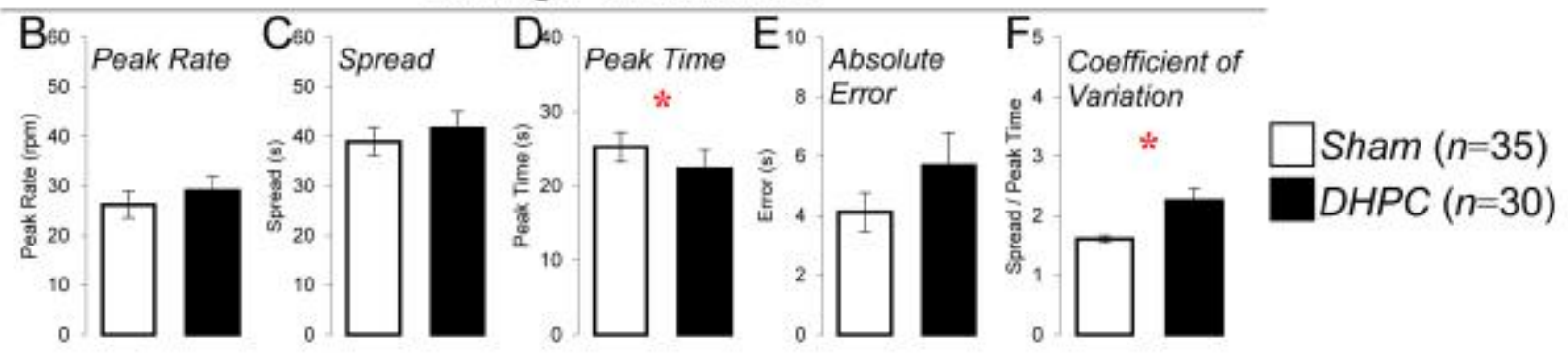


Figure 4 .
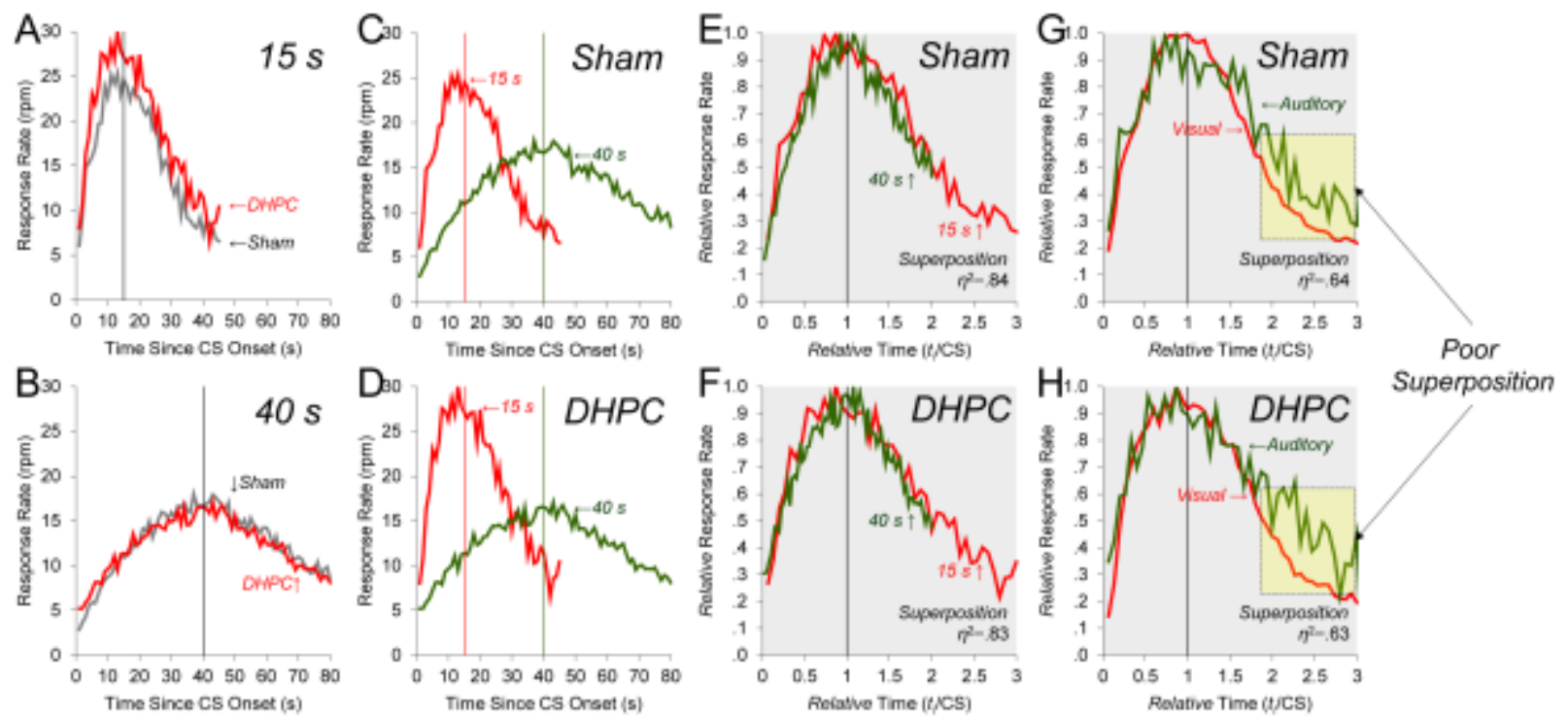
Figure 5.

15-s vs. 40-s CS (Sham)
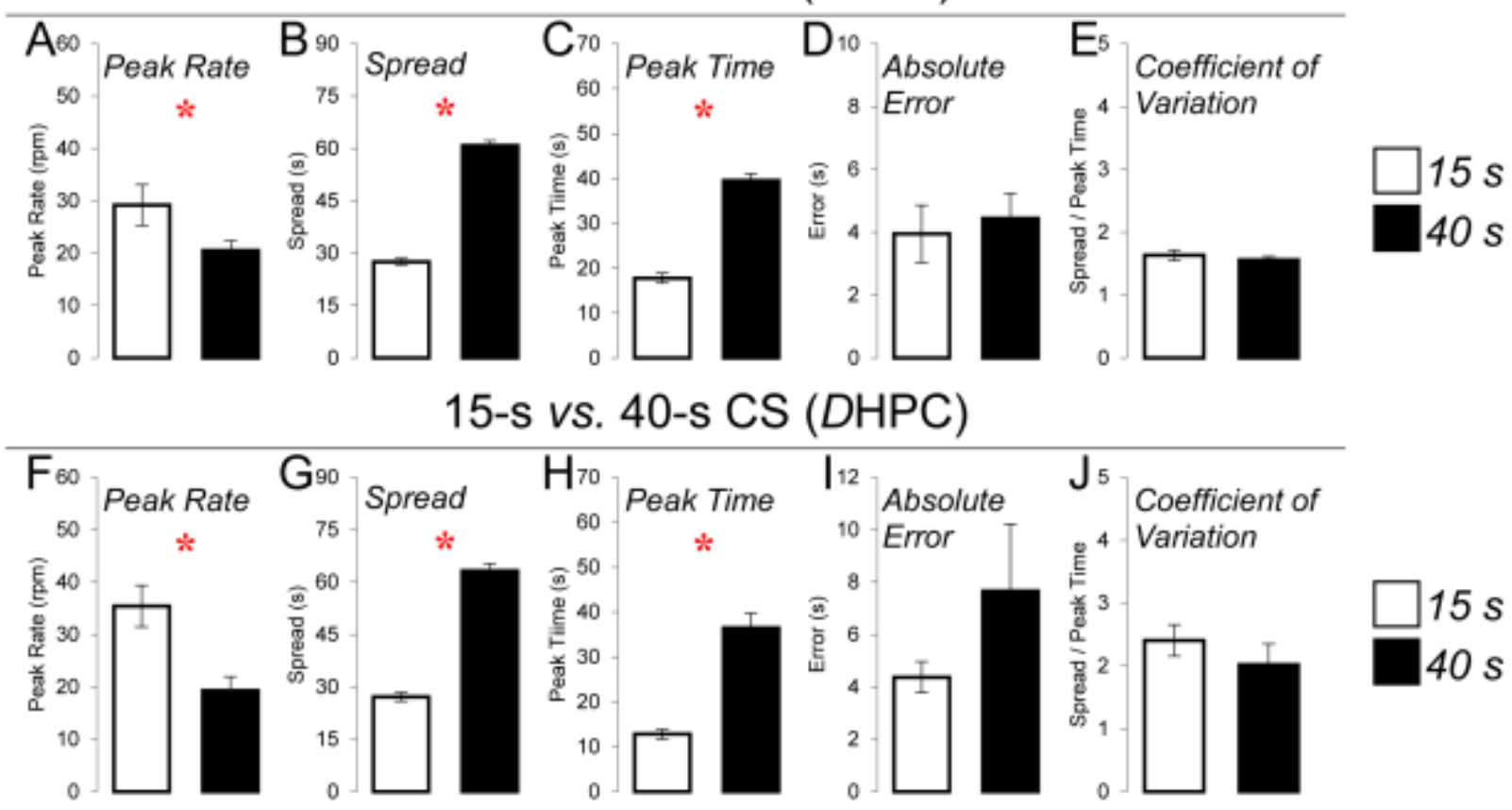
Figure 6.

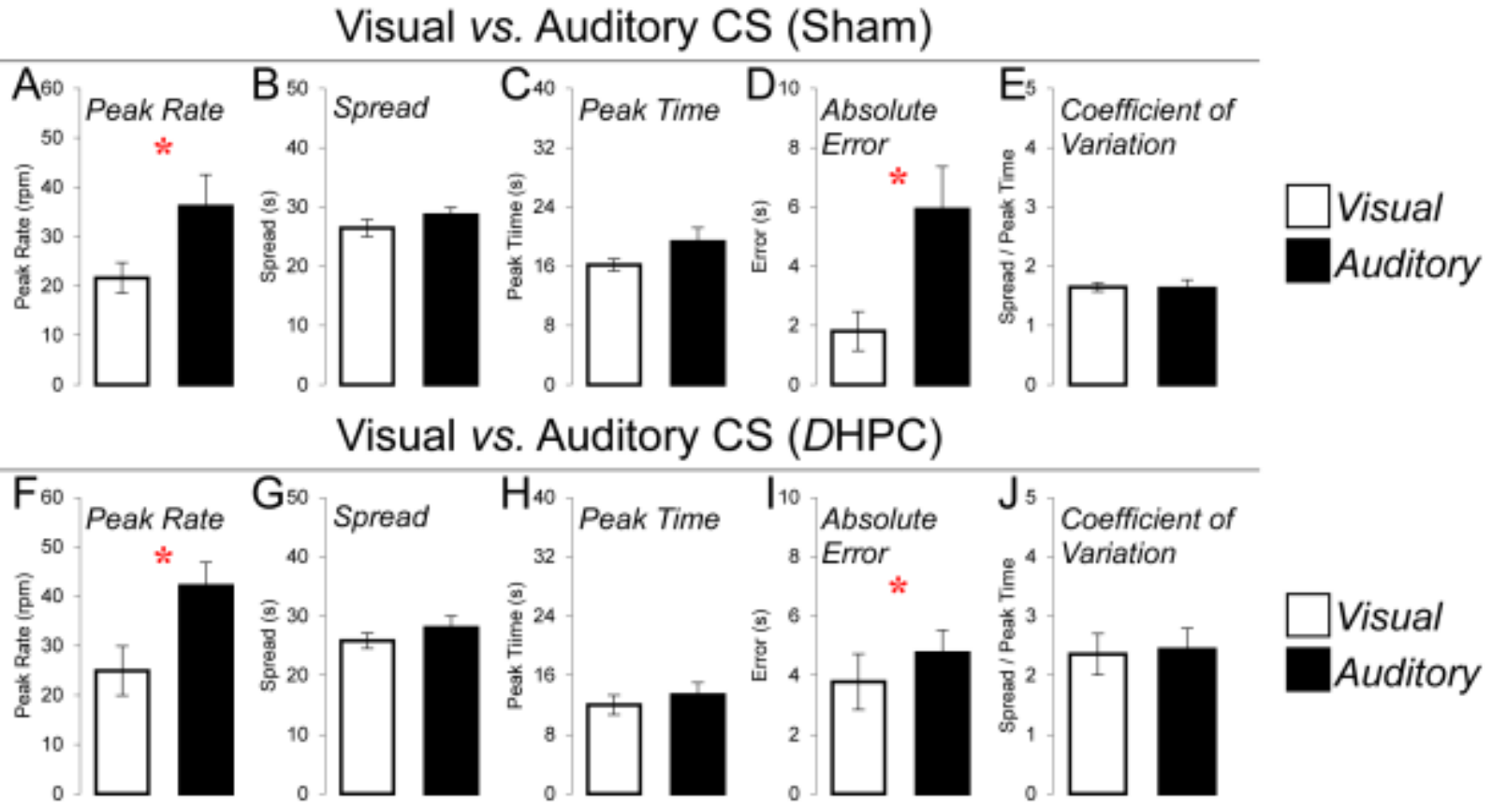


Figure 7.
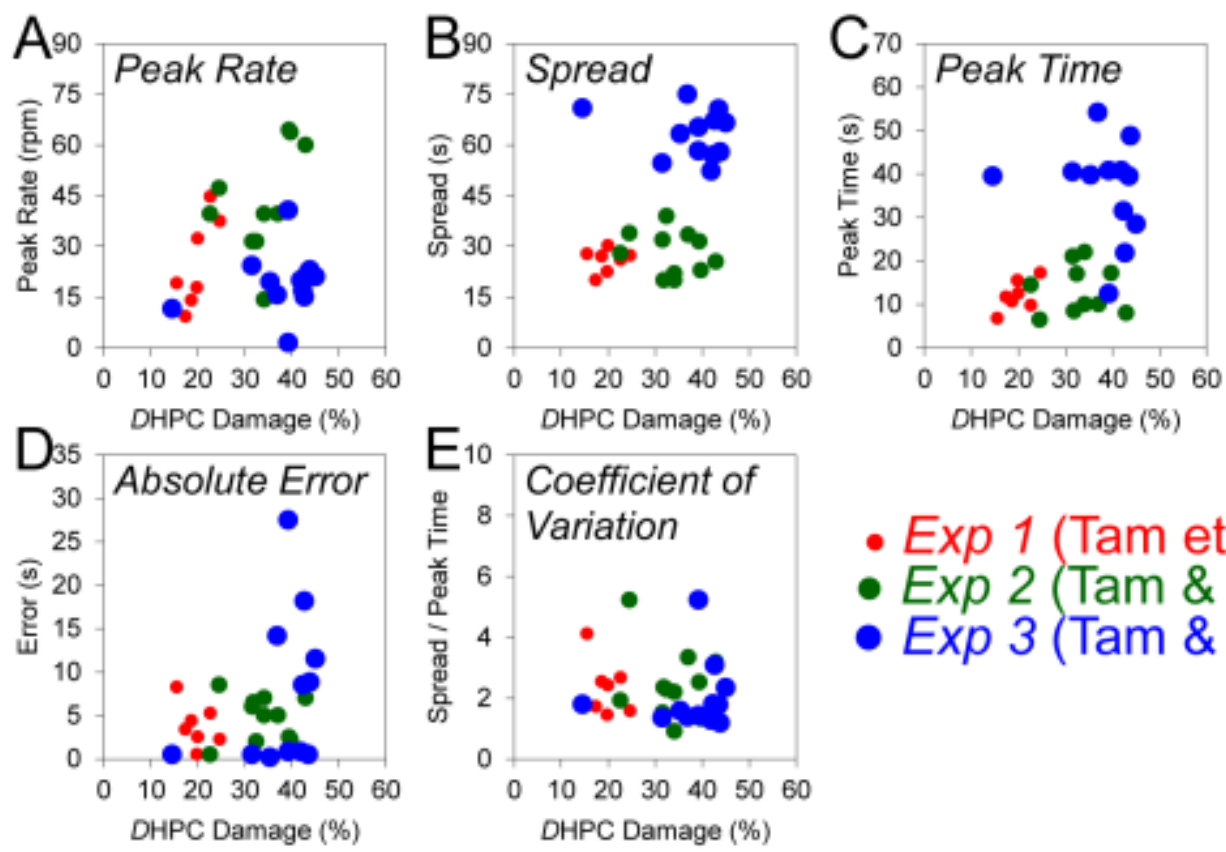

- Exp 1 (Tam et al. 2013)

- Exp 2 (Tam \& Bonardi 2012a)

- Exp 3 (Tam \& Bonardi 2012b) 
Figure 8.

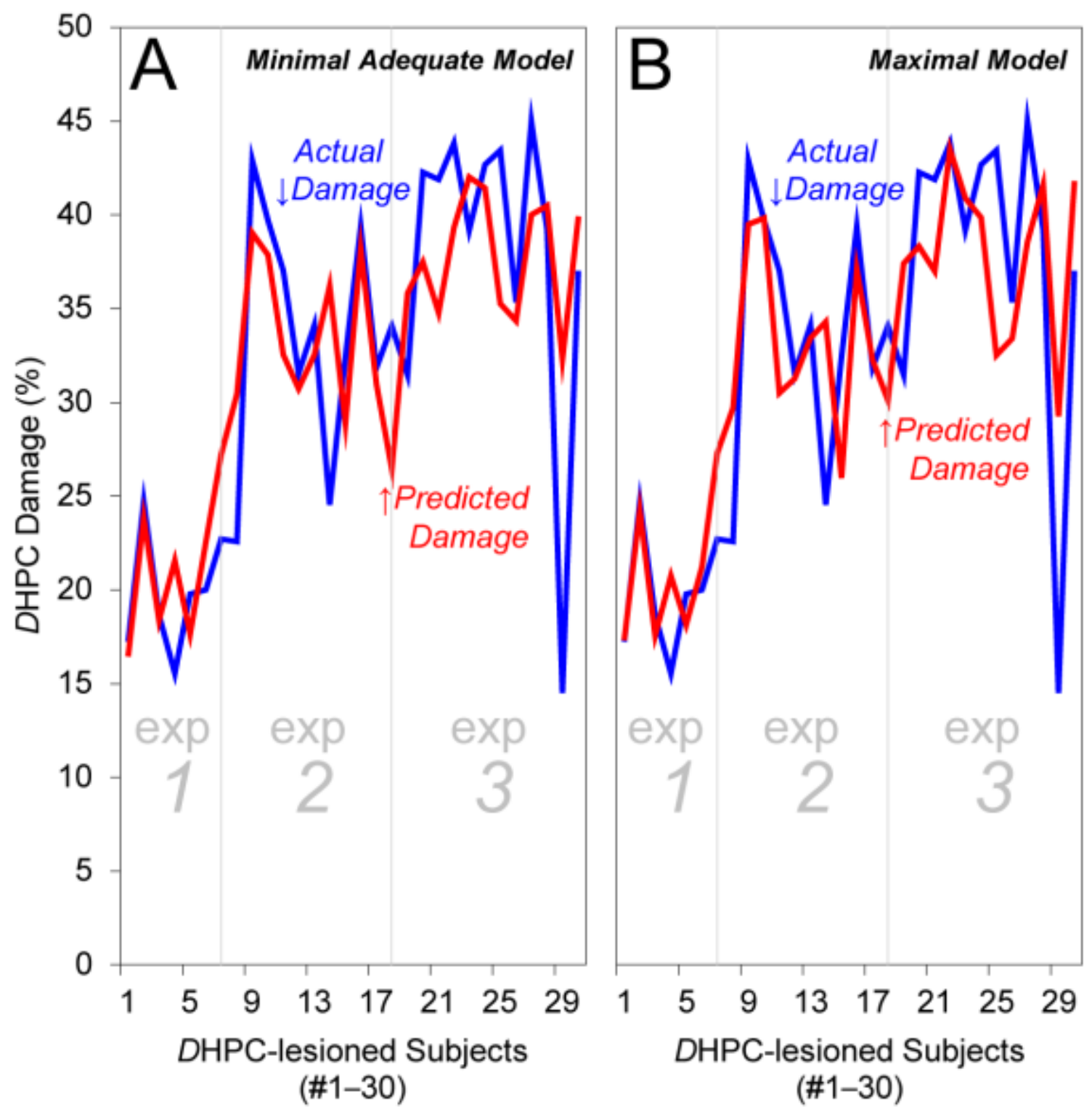


Figure 9.
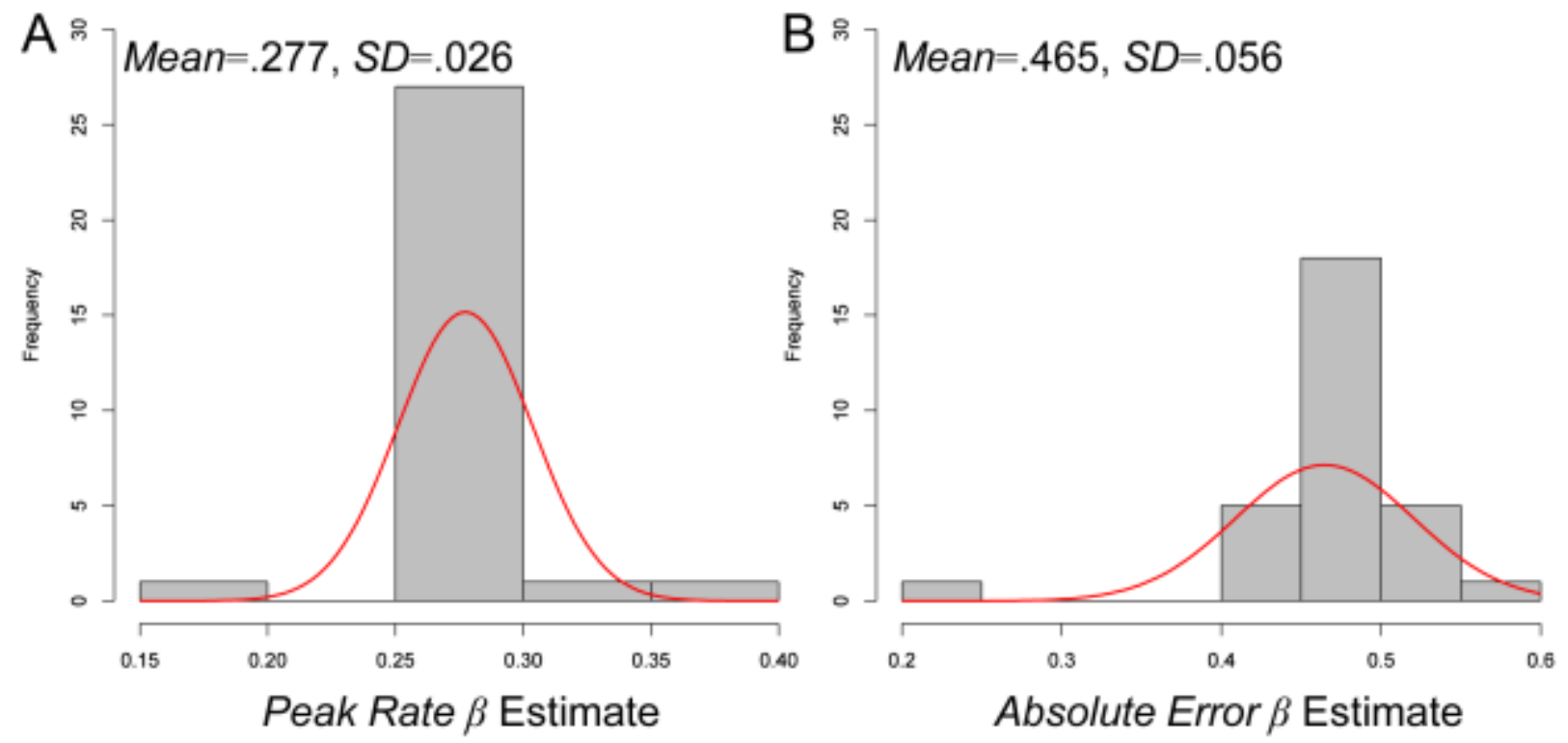